\title{
INGUINAL HERNIOPLASTY ACCORDING TO LOTHEISSEN AND McVAY
}

\author{
Zbyněk Vobořil \\ Charles University in Prague, Faculty of Medicine in Hradec Králové: Department of Surgery \\ Summary: Inguinal hernioplasty represents one of the most frequently performed operation in surgery. In the article the \\ author describes the technique of inguinal hernioplasty according to Lotheissen and McVay. He has an excellent expe- \\ rience when using this procedure. This communication tried to focus the attention of surgical community on this met- \\ hod.
}

Key words: Inguinal hernioplasty; Inguinal hernia

Inguinal hernioplasty represents one of the most frequently performed surgical interventions, being only second in frequency after appendectomy. In spite of the fact that hernioplasty is frequently performed by surgical residents, this does not mean that treating patients with hernias is an easy task, as we often realize when encountered with recurrence. Current surgical practice stresses the importance of meticulous reconstruction of deep inguinal layer. This is by no means a new concept. Back in 1804 Cooper suggested that transversal fascia discovered by him protects against hernias. It was not until 1941 that McVay and coworkers proved on preparations that transversal fascia is not attached to inguinal, but to pectineal (Cooper's) ligament (Fig. 1). He noticed that when transversal fascia is attached to Cooper's ligament there may be no reason for disturbing this spatial relationship during inguinal reconstruction, and there is no reason joining deep structures and fascia to inguinal ligament. By proposing the procedure McVay essentially rediscovered and made popular an older technique discribed by Lotheissen. Georg Lotheissen, an Austrian surgeon, was the first to use Cooper's ligament for repair of inguinal hernia in 1898. He discovered an effective method of reconstructing posterior wall of inguinal canal which plays an important role in the origin and treatment of inguinal hernias. The technique, most often referred to as McVay's method, which should rather be called technique after Lotheissen and McVay, slipped into oblivion and is currently not widely used.

At the Department of Surgery of Faculty of Medicine and Teaching Hospital we are using the technique presented in cases of important defects of the posterior wall of inguinal canal, and in surgical management of recurrent hernias, including femoral hernias.

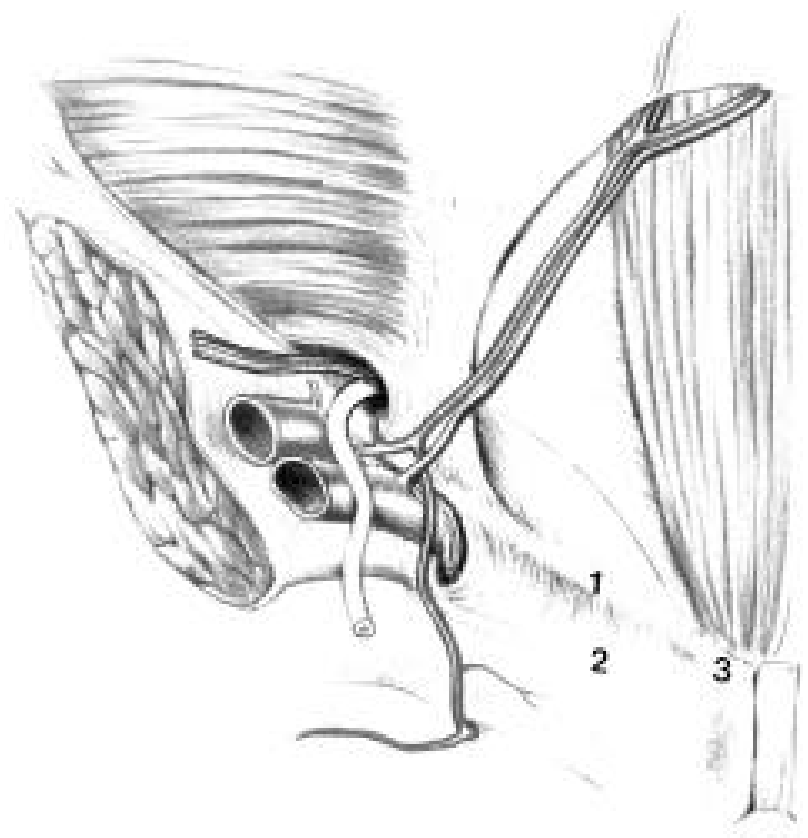

Fig. 1: View of lower abdominal wall quadrant from inside. Transversal fascia attaches to Cooper's ligamentum. 1 - lig. inguinale, 2 - lig. pectinale Cooperi, 3 - tuberculum pubicum.

\section{The procedure}

The procedure requires exact identification of anatomical structures and meticulous work. The procedure is identical to standard techniques up to the moment of closing the inguinal sac. Anulus inguinalis internus is contracted and then moved laterally. 


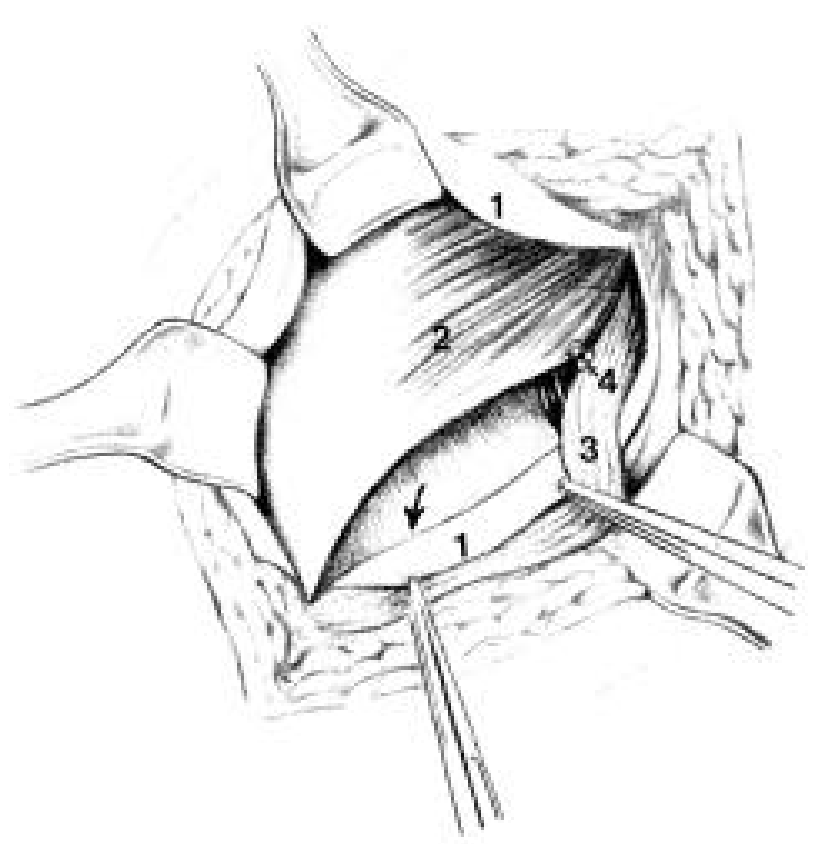

Fig. 2: Inguinal hernioplasty according to Lotheissen and McVay: preparation behind inguinal ligament caudally (arrow). 1 - aponeurosis m. obliqui abdominis ext., 2 - m. obliquus abdominis internus, 3 - funiculus spermaticus, 4 - rest of the hernial sac (ligated).

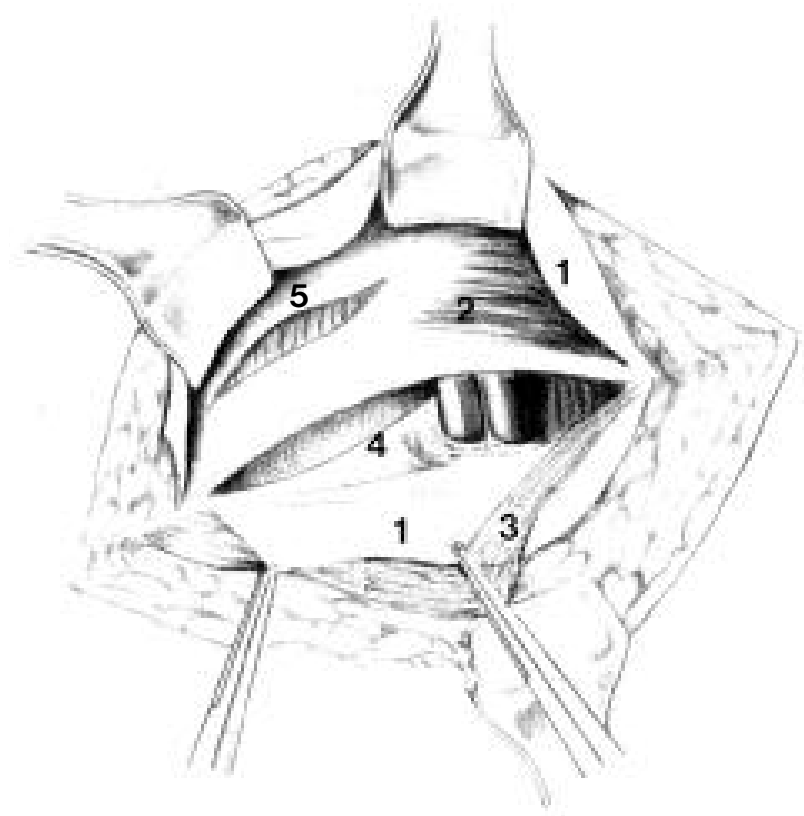

Fig. 3: Inguinal hernioplasty according to Lotheissen and McVay: by preparation behind inguinal ligament caudally ramus superior and pecten ossis pubis with Cooper's ligament are made free. 1 - aponeurosis $\mathrm{m}$. obliqui abdominis ext., 2 - m. obliquus abdominis internus, 3. - funiculus spermaticus, 4 - lig. pectineale Cooperi, 5. - Hockeystockschnitt.

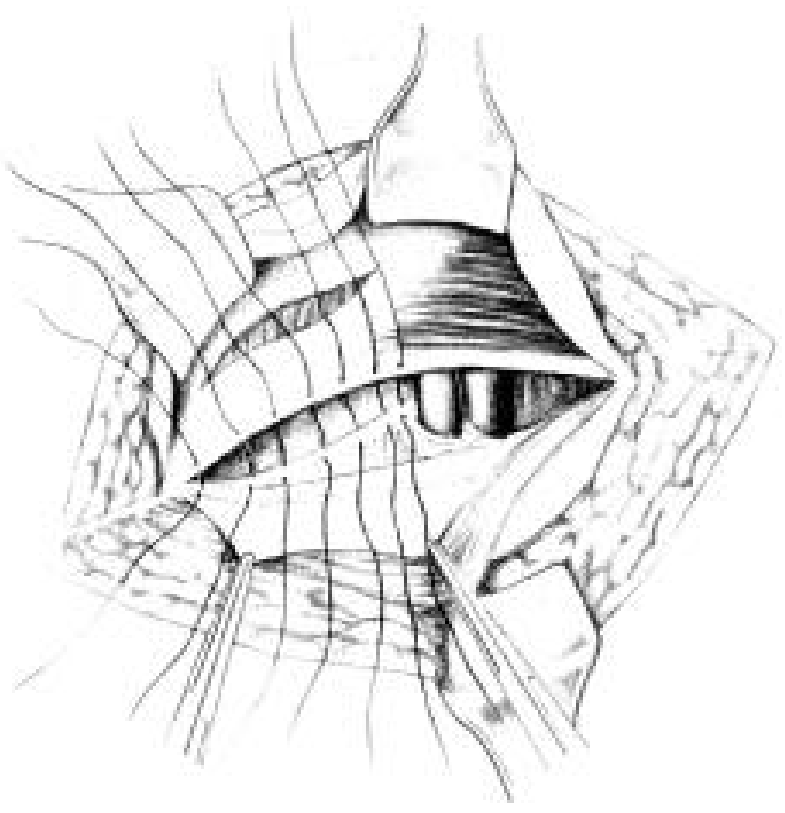

Fig. 4: Inguinal hernioplasty according to Lotheissen and McVay: hernioplasty is performed by suturing, superiorly, transversal fascia medially up to tendon conjoint, inferiorly to pectineal ligament and medially to tuberculum pubicum. The most lateral suture is placed in close proximity and medially to femoral vein.

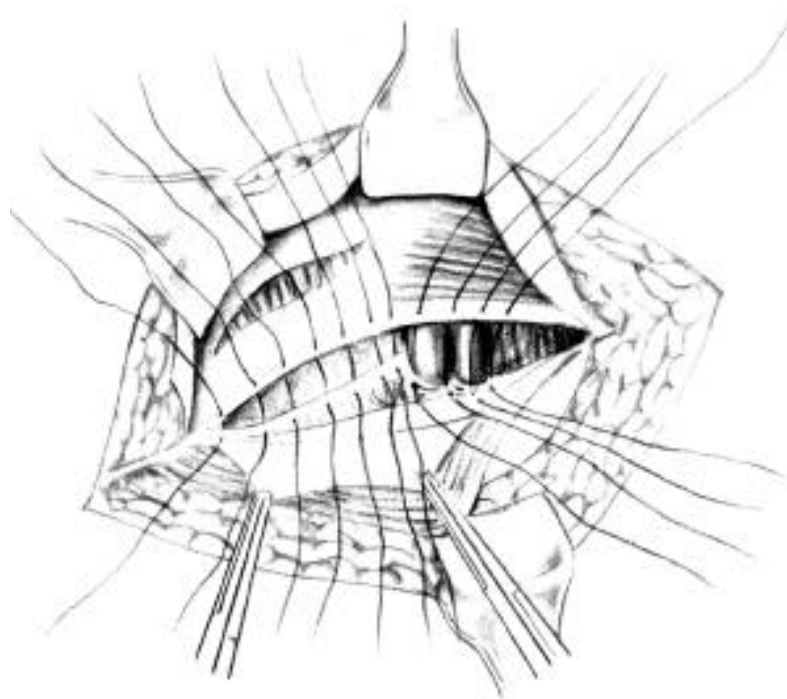

Fig. 5: Inguinal hernioplasty according to Lotheissen and McVay: in the region above femoral vessels 2-3 sutures between fascia transversalis and connective tissue around femoral vessels, Thompson's band or deep inferior layer of inguinal ligament are placed (transient suture). 


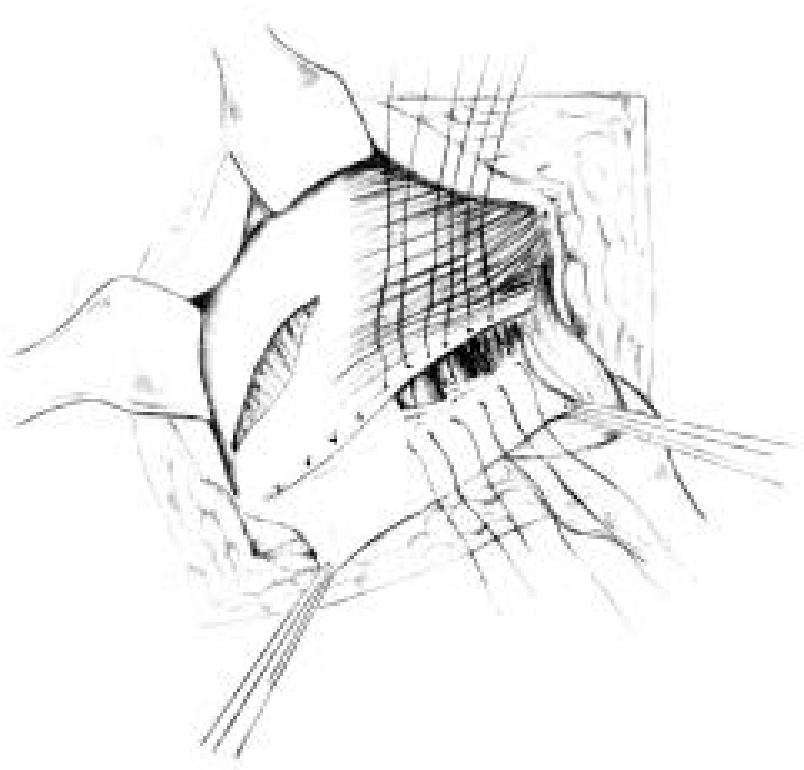

Fig. 6: The reconstruction of deep inguinal layer according to Lotheissen and McVay.

By preparation behind inguinal ligament caudally ramus superior and pecten ossis pubis with Cooper's ligament are made free (Fig. 2 and 3). Hernioplasty is performed by suturing, superiorly transversal fascia medially up to tendon conjoint, inferiorly to pectinal ligament and medially to tuberculum pubicum (Fig. 4). The most lateral suture is placed in close proximity and medially to femoral vein under careful visual control. In the region above femoral vessels we place 2-3 sutures between transversal fascia and connective tissue around femoral vessels, Thompson's band or deep inferior layer of inguinal ligament (Fig. 5). This part of the suture - transient suture - was considered by McVay to be the weakest point of the hernioplasty, and the line of the suture is moved here one layer more superficially to inguinal ligament.

In addition, a relaxing incision of both posterior sheaths of rectal muscle is performed (so called Hockeystockschnitt - Fig. 3-6), which makes possible performing the deep suture without tension.

Above the structure thus created the spermatic funicle is placed, and cremaster muscle is resected, followed by a suture of the exterior oblique muscle fascia to inguinal ligament.

\section{Patients}

Between 1974 and December 1996 this procedure of hernioplasty after Lotheissen and McVay was performed at the Department of Surgery in Hradec Králové in 225 cases. Long term results were evaluated in a cohort of 32 patients ( 29 males and 3 females, aged 21 to 74 years). The most numerous group of patients consisted of those with direct inguinal hernia (16 cases), indirect inguinal hernia was present in 10 cases, femoral hernia in 2 cases, a recurrent femoral hernia was present in 4 cases. The patients were followed for 2 to 19 years after surgery.

Among these 32 patients recurrence was observed in one case of a 65 year old man treated for recurrent direct inguinal hernia. Postoperative course was complicated by bronchitis with massive expectoration. The recurrence was diagnosed 9 months after surgery. A reoperation was performed and the patient is recurrence-free after 8 years.

\section{Conclusions}

Inguinal hernia can be managed successfully using a variety of methods based on the type, extent and experience of the surgeon. A technique should be chosen, however, which pays attention to meticulous reconstruction of deep inguinal layers. The method according to Lotheissen and McVay is one of these procedures. At our institution we made an excellent experience using this procedure, and this communication tried to focus the attention of surgical communy on this method.

Figures were drawn by Josef Bavor, Ph.D.

\section{References}

1. Cooper A. In: Hyrtl J: Handbuch der topographischen Anatomie, 3. Auflage, Wien: W. Braumuler, 1857

2. Lotheissen G. Radikaloperation der Schenkelhernien. Zbl Chir 1898;25:548-55. In: Kole W. Operationen an der Bauchdecke und bei Unterleibsbruchen, BierBraun-Kümmel Chiurgische Operationslehre, Leipzig: J.A. Barth, 1972

3. McVay ChB. Ann Surg 1941;113:1111-12. In: McVay ChB, Anson BJ. Inguinal and femoral hernioplasty. Surg Gynecol Obstet 1949;88:473-85.

Submitted December 1998

Accepted April 1999

Prof. MUDr. Zbyněk Vobořil, DrSc., Charles University in Prague, Faculty of Medicine in Hradec Králové, Department of Surgery, 50005 Hradec Králové, Czech Republic. 